\title{
APRENDER A CONSTRUIR PROYECTOS PROFESIONALES Y VITALES
}

\section{LEARN HOW TO BUILD PROFESSIONAL AND VITAL PROJECTS}

\author{
Soledad Romero Rodríguez* \\ Facultad de Ciencias de la Educación - Universidad de Sevilla
}

\begin{abstract}
RESUMEN
No cabe duda de que el entorno sociolaboral está sometido a constantes cambios. Para que la persona pueda hacerles frente es preciso que aprenda a tomar decisiones autónomas y conscientes y construir proyectos profesionales y vitales. En este artículo presentamos los contenidos que deberían contemplarse en una intervención orientadora cuya finalidad fuera precisamente fomentar dicho aprendizaje.
\end{abstract}

Palabras clave: Proyecto profesional y vital — toma de decisiones- proceso de orientación profesional.

\begin{abstract}
There is no doubt than social and labour environment is always changing. In order people face these changes is necessary that they learn to make autonomous decisions and elaborate career and vital plans. In this article we present the contents which should be considered in a process of guidance which principal aim where precisely to facilitate this kind or learning.
\end{abstract}

Key words: Career and vital plans or projects — decision making — career guidance process

\section{Introducción}

Nos encontramos inmersos en una sociedad sometida a constantes y acelerados cambios. En este contexto se hace necesario que la persona cuente las herramientas necesarias para poder construir y salvaguardar su identidad personal y profesional, en equilibrio con los determinantes sociales, económicos y culturales. El proceso de orientación debe facilitar

* Profesora Titular de Universidad en la Facultad de Ciencias de la Educación de la Universidad de Sevilla. Sus publicaciones e investigaciones han girando en torno a la orientación para la transición de la escuela a la vida activa, el diseño de programas de orientación y la orientación profesional desde una perspectiva educativa y crítica. En la actualidad participa en proyectos de investigación relacionados con el desarrollo de competencias profesionales y la orientación universitaria. 
el aprendizaje del proceso de toma de decisiones conscientes y motivadas y de construcción de proyectos de acción coherentes con dichas decisiones.

Por otro lado, la sociedad actual reclama un concepto de profesionalidad según el cual la persona ha de estar dotada de competencias transferibles o clave que tienen que ver con la toma de decisiones, la resolución de problemas, la autonomía, el trabajo en grupo, la planificación de su propio trabajo (Alex, 1991; Bunk, 1994; Echeverría, 1993, 1999, 2002) ... A la vez, se reivindica la necesidad de compatibilizar el ejercicio profesional con otras actuaciones y situaciones de carácter personal, familiar, social y cultural.

Esta realidad justifica la necesidad de desarrollar sistemas y programas de orientación que faciliten el aprendizaje del proceso de construcción de proyectos profesionales y vitales. En este artículo analizaremos, en primer lugar, el concepto de proyectos profesional y vital, para pasar a continuación a describir los contenidos a desarrollar en la acción orientadora que se plantee como meta la facilitación del aprendizaje de su proceso de construcción.

\section{Concepto y características de Proyecto profesional y vital}

El análisis de algunas de las definiciones de proyecto profesional y vital, nos ayudarán a delimitar los rasgos esenciales que lo caracterizan.

"Se trata de interrogarse sobre la propia vida, sobre el sentido que se desea darle. Interrogarse sobre su vida, vida en movimiento, movimiento alimentado, sostenido por un ideal, un objetivo, un proyecto; ser en proyecto, más que tener un proyecto" (Bernard, 1995:36).

"El proyecto, por consiguiente, puede definirse como un poner en relación, de modo significativo, el pasado, el presente y el futuro, quedando privilegiada la última dimensión" (Guichard, 1995:17).

“... aceptar el proyecto como intención, aspiración a ser u a hacer, como una trayectoria hecha de líneas derechas y vueltas, de continuidad y de rupturas, de fracasos y éxitos, de influencias múltiples, personales, colectivas, ambientales, de estrategias pensadas y de azar, de oportunidades a aprovechar. Por tanto, el proyecto puede abrir, dar un sentido, coherencia, contribuir a la creación de un ser en devenir, enraizado en un pasado, un presente y un futuro colectivo" (Mure, 1997:33).

"Para una persona perteneciente a un grupo o a una sociedad, el proyecto es un compromiso permanente entre sus aspiraciones, sus intereses, sus sistemas de valores y de representaciones a los que se refiere continuadamente y los medios de que dispone, las estructuras sociales de las que depende, la manipulación de que es objeto y las posibilidades de cambio que le permitirán modificar su situación." (Rodríguez Moreno, 1999:16).

"El proyecto

- Es una construcción activa,

- En la que se trabaja 'sobre' y se expresa la propia imagen de sí mismo/a,

- Que implica la adquisición de una serie de conocimientos, habilidades y actitudes,

- Que requiere de un proceso previo de información/exploración/decisión/formulación de objetivos, 
- Que se concreta en un plan de acción,

- Y genera desarrollo personal.

- Es posible generar y favorecer este proceso de aprendizaje" (Romero Rodríguez, 1999:40).

A partir de estas definiciones, se puede considerar que los rasgos que definen a los proyectos profesionales y vitales son los siguientes (Fig. 1):

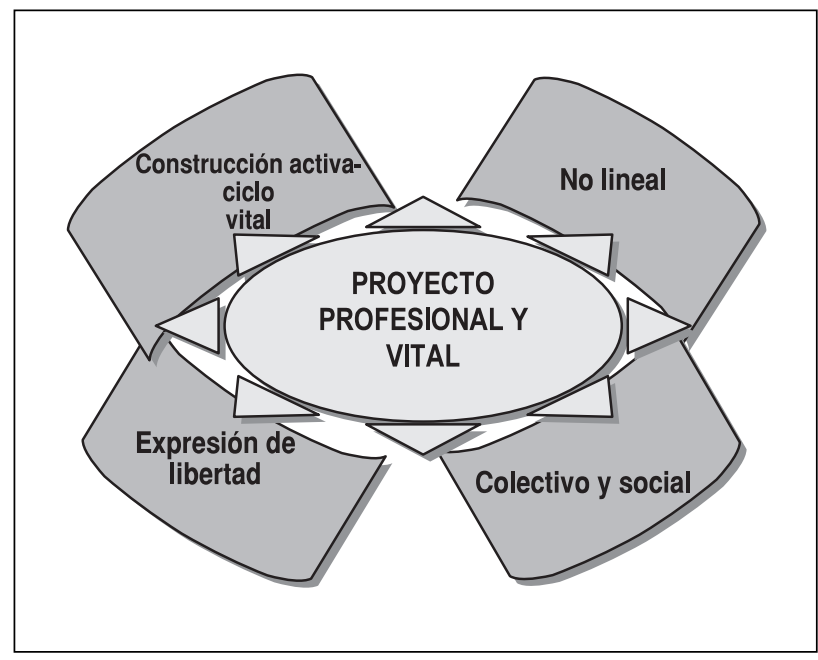

FIGURA 1.

Rasgos proyecto profesional y vital.

- Es una construcción activa que se desarrolla a lo largo de todo el ciclo vital. El proyecto profesional y vital supone que la persona esté en un constante estado de búsqueda de sentido de su propia vida: su forma de ser, las situaciones de su entorno, la manera de relacionarse con su contexto, la incidencia de su pasado, lo que desea y/o espera que sea su futuro...

Por tanto, el proyecto no se realiza en un momento puntual y para toda la vida. Como señala Bernard (1995) la persona es un "ser en proyecto". Es decir, constantemente está cuestionándose su vida: se trata de comprender el presente, poniéndolo en relación con el pasado, con la finalidad de preparar el futuro.

Pero ¿qué supone este "preparar el futuro"? Construir un proyecto profesional y vital implica buscar un estilo de vida, el cual será el hilo conductor, el eje sobre el que se articulará la conjugación de los diferentes roles a desempeñar a lo largo del ciclo vital. Es decir, el proyecto profesional y vital va a facilitar la búsqueda del equilibrio entre esos diferentes roles (madre-profesional; trabajo-ocio...) y, por tanto, la resolución positiva de los conflictos que pueden aparecer entre ellos, dando coherencia a la propia trayectoria vital. 
Por otra parte, el proyecto profesional permite que la forma de desempeñar los roles (acción) a los que se está haciendo referencia tengan coherencia con los propios valores personales y profesionales (hay muchas formas de ser profesor o profesora, por ejemplo).

- Se trata de un proceso no lineal, que se concreta en un plan de acción abierto a las oportunidades que va ofreciendo el contexto. Contrariamente a lo que se pueda pensar en principio al escuchar la palabra "proyecto", éste no encorseta a la persona, sino que la pone en situación de alerta, de constante exploración e indagación, la prepara para poder captar cualquier oportunidad que ofrezca el entorno y analizarla inmediatamente para valorar si responde o no a las propias necesidades, a los propios valores. Este estado de constante alerta es intencional, es decir, implica una disposición a querer comprender, afrontar y enfrentarse a las condiciones del entorno que están en constante cambio y dentro de las cuales se tiene que desarrollar la trayectoria profesional y vital. Como señala Fernández Sierra (1998), este estado de constante cuestionamiento respecto a lo que ocurre a nuestro alrededor debe facilitar una valoración crítica de estas situaciones desde posicionamientos éticos de carácter individual y colectivo.

La idea, por tanto, es que es necesario que la persona adopte una actitud consciente e intencional de apertura en relación a las situaciones y acontecimientos inesperados, a los cuales tratará de buscar sentido tomando como referencia sus propios valores, sus propias necesidades, que habrán sido previamente analizados. Sólo a partir de este proceso de valoración de las oportunidades, podrá aprovechar el potencial de estas situaciones e integrarlas como parte de su propio proyecto profesional.

Esta idea está en la base de diversos conceptos que están apareciendo en los últimos años: Oportunismo vocacional (Pelletier, 1994); Planned happenstance (oportunidad planificada) (Mitchell, Levin y Krumboltz, 1999); Planful serendipity - realización de descubrimientos inesperados que se integran en un plan flexible que permite que la persona provoque y/o aproveche dichos descubrimientos- (Watts, 1999).

- Es una expresión de libertad. El estado de constante búsqueda de sentido, permite a la persona tomar conciencia de los determinismos que inciden en su trayectoria profesional y vital, lo que facilita la búsqueda de los medios para superarlos y/o adaptarse críticamente a ellos. Para Boutinet (1990) la construcción de los proyectos profesionales y de vida debería ayudar a la persona a relativizar los estereotipos y a potenciar las capacidades que facilitan la autonomía personal.

- Tiene un carácter colectivo y social en varios sentidos:

a) El proceso de construcción del proyecto se desarrolla a partir de la vivencia de experiencias, de situaciones que se dan en un contexto social determinado;

b) Se facilita este proceso de construcción a través del trabajo en grupo, en el que se comparten las experiencias, puntos de vista, que ayudan a comprender, a encontrar el sentido de la situación personal por parte de cada individuo;

c) La toma de conciencia colectiva de los determinismos, los obstáculos que dificultan el desarrollo personal y profesional, puede ayudar a planificar acciones de lucha frente a dichos condicionantes. 


\section{Razones para aprender a construir Proyectos profesionales y vitales}

Son variados los motivos que justifican la necesidad de aprender a construir proyectos profesionales y vitales y que ésta sea la principal finalidad de los procesos de orientación. Estas justificaciones encuentran diversas fuentes de fundamentación:

1. Situación sociolaboral, caracterizada por los constantes e imprevisibles cambios, la flexibilización del mercado de trabajo, la inseguridad en el puesto de trabajo, el desempleo, la exigencia de nuevas, polivalentes y variadas competencias profesionales, las nuevas formas de trabajo... (Castells, 1994, 1996; Caspar, 1994; CEE, 1994, 1996; Delors, 1996; Giarini y Liedtke, 1998...) Todo ello hace que las trayectorias profesionales sean más complejas, paradójicas e inciertas. Por tanto, se hace necesario que la persona esté dotada de los instrumentos y las herramientas que le permitan desenvolverse en este contexto sin menoscabo de su desarrollo integral, a la vez que se debe fomentar el compromiso con una acción encaminada a mejorar las condiciones de vida.

2. Las propias características del que se considera el objeto de la orientación profesional: el desarrollo de la carrera. Desde distintos planteamientos teóricos, se considera que la carrera está definida por su carácter evolutivo - a lo largo de todo el ciclo vital- (Super, 1990, 1995; Watts, 1999), subjetivo - la carrera como percepción personal- (Arnold y Jackson, 1997); complejo - no-lineal, confluencia de roles diversos - (Boursier y Langlois, 1993; Collin y Watts, 1996; Fernández Sierra, 1998; Mure, 1996; Pelletier, 1994), paradójico — conflictos de roles, contradicciones personales y contextuales - (Bousier y Langlois, 1993; Corominas e Isús, 1998; Law, 1999; Savickas, 1993; soláis, 1993, 1998); contextual — desarrollo personal en relación dialéctica con el desarrollo social- (Collin, 1997; Herr, 1996; Riverin-Simard, 1996; Vondraceck y col., 1986; Young, 1984; Young, Valach y Collin, 1996, colectivo - optimización del desarrollo personal y social a través de la acción participativa y cooperativa- (Collin, 1997; Defrenne, 1998; Latreille, 1984; Mure, 1997).

3. Las características del proyecto profesional y vital, descritas en el apartado anterior, hacen que éste se constituya en elemento básico de comprensión, dominio y/o transformación de las situaciones así como de autoría del propio desarrollo personal y profesional. Asimismo, es un elemento que propicia la acción colectiva transformadora.

\section{Proceso y contenidos de construcción de Proyectos profesionales y vitales}

La construcción de proyectos profesionales y vitales es un proceso intencional en el que se tiene una actitud constante de cuestionamiento, gracias al cual se puede realizar un ejercicio de anticipación del futuro (intención), que se plasmará en un plan de acción que estará marcado por la constante evaluación de la validez de los objetivos a los que pudiera apuntar en un principio el proyecto (carácter no lineal del proyecto). 
Como señala Guichard (1995) el proyecto es diferente a lo que "quiero hacer" o lo que "me gustaría hacer", e implica: Valorar la espera presente (¿merece la pena el esfuerzo a realizar? ¿hasta cuándo puedo esperar? ¿qué puedo hacer mientras espero?); cuestionar los medios necesarios para conseguir los objetivos (¿están disponibles? ¿son acordes con mis valores?); valorar la validez futura de las intenciones presentes (¿seguirán siendo importantes en un futuro?).

El desarrollo de este proceso de forma sistemática e intencional implica la puesta en práctica de las diferentes tareas del desarrollo vocacional (exploración, cristalización, especificación y realización) las cuales se integran en los tres tipos de cultura que deben desarrollarse desde una perspectiva crítica de la orientación profesional: cultura de la anticipación, cultura del proyecto, cultura de la acción.

En el esquema siguiente se representan los contenidos que están implicados en el proceso de construcción de proyectos profesionales y vitales y la relación entre ellos. De este esquema se deriva el tipo de contenidos a trabajar a través de la intervención orientadora profesional, si bien todos ellos no tienen que ser trabajados necesariamente a un tiempo y en una sola acción orientadora. La selección de los contenidos a trabajar dependerá de las necesidades específicas de las personas destinatarias, el ámbito de actuación y las posibilidades reales de actuación.

\begin{tabular}{|c|c|c|}
\hline \multirow[t]{2}{*}{ Aprender a anticiparse } & Aprender a explorar (Exploración) & $\begin{array}{l}\text { Sí mismo/a } \\
\text { Entorno }\end{array}$ \\
\hline & \multicolumn{2}{|c|}{ Construir identidad personal y colectiva (Cristalización) } \\
\hline \multirow{2}{*}{$\begin{array}{l}\text { Aprender a construir } \\
\text { proyectos }\end{array}$} & \multirow{2}{*}{\multicolumn{2}{|c|}{$\begin{array}{l}\text { Aprender a tomar decisiones (Especificación } \\
\text { Aprender a elaborar un plan (Realización) }\end{array}$}} \\
\hline & & \\
\hline Aprender a actuar & \multicolumn{2}{|c|}{$\begin{array}{l}\text { Desarrollo empleabilidad y ocupabilidad (Realización) } \\
\text { Desarrollo de compentencias participativas (Realización) } \\
\text { Planificación del ocio y el tiempo libre (Realización) }\end{array}$} \\
\hline
\end{tabular}

En la figura 2 se recogen de forma sintética los tipos de aprendizajes que se deben realizar para poder construir proyectos profesionales y vitales. Estos contenidos no se integran en un proceso lineal, sino recurrente, en espiral.

\section{Aprender a anticiparse}

Los conocimientos, habilidades y actitudes vinculadas a este aprendizaje están relacionados con las tareas de exploración y de cristalización. En el cuadro 1 se recogen algunas de las habilidades y actitudes que deben ser desarrolladas para que se puedan dominar dichas tareas: 


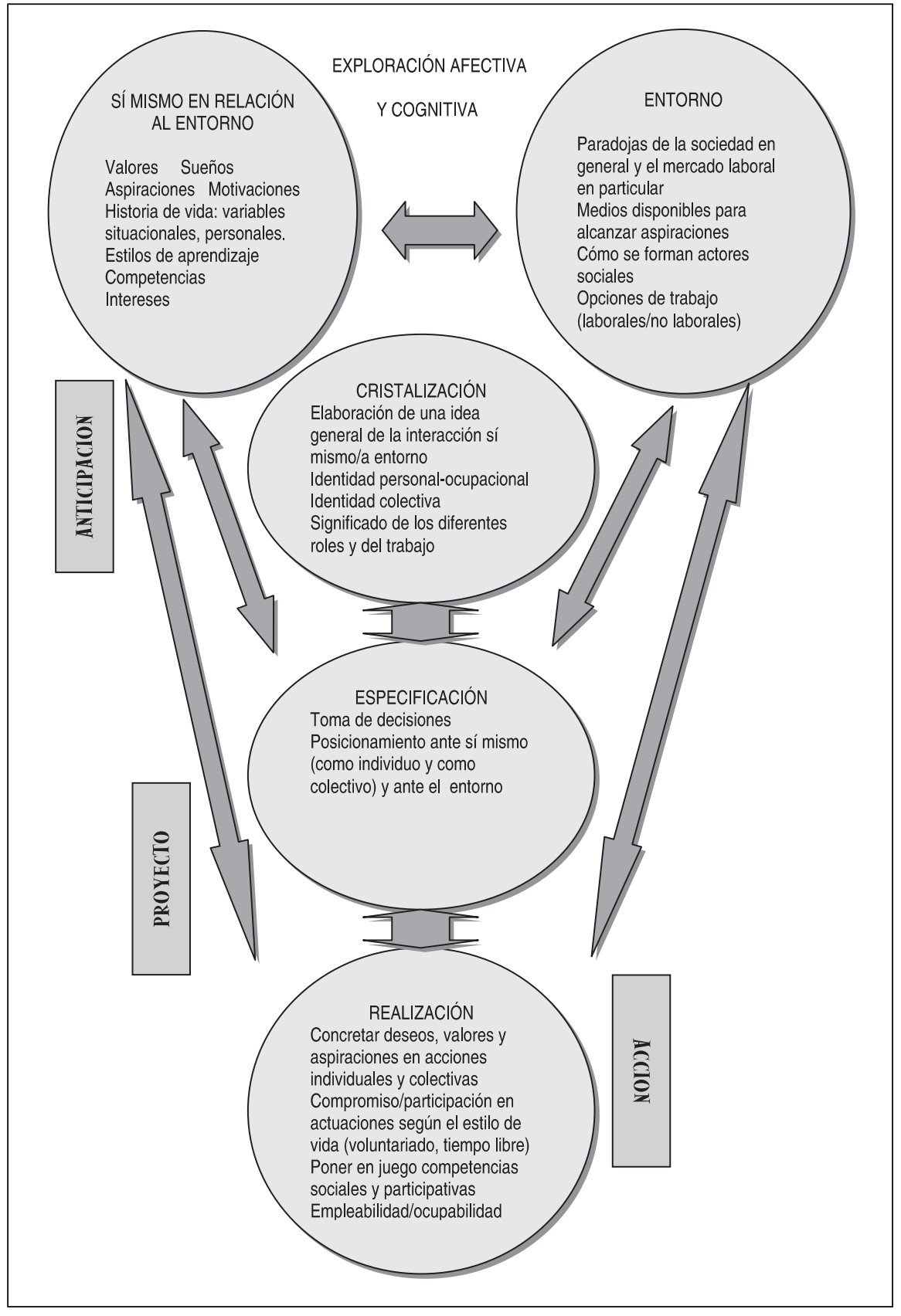

FIGURA 2.

Áreas de la acción orientadora profesional. 


\section{CUADRO 1: Habilidades y actitudes implicadas en la construcción de proyectos profesionales y vitales (adaptado de Pelletier y Bujold, 1984).}

\begin{tabular}{|c|c|c|}
\hline $\begin{array}{c}\text { Tareas de desarrollo } \\
\text { vocacional }\end{array}$ & Habilidades & Actitudes \\
\hline Exploración & $\begin{array}{l}\text { Observar } \\
\text { Describir } \\
\text { Preguntar } \\
\text { Descubrir } \\
\text { Definir } \\
\text { Imaginar } \\
\text { Reducir } \\
\text { Asociar }\end{array}$ & $\begin{array}{l}\text { Apertura } \\
\text { Sensibilidad } \\
\text { Curiosidad } \\
\text { Tolerancia } \\
\text { Imaginación } \\
\text { Elaboración de hipótesis } \\
\text { Interesarse } \\
\text { Autoestima }\end{array}$ \\
\hline Cristalización & $\begin{array}{l}\text { Reagrupar } \\
\text { Clasificar } \\
\text { Resumir }\end{array}$ & $\begin{array}{l}\text { Organización } \\
\text { Orden } \\
\text { Coherencia } \\
\text { Continuidad }\end{array}$ \\
\hline
\end{tabular}

A continuación se analizan los aspectos específicos de estos contenidos.

\section{Aprender a explorar}

El dominio de la tarea de exploración supone el desarrollo de la capacidad para poder experimentar, recoger, vivir todas aquellas situaciones, experiencias, oportunidades, informaciones, sentimientos que ayudarán a:

a) Construir una imagen más ajustada de sí mismo/a y, por tanto, mejorar la autoestima;

b) Elaborar una representación propia del entorno social y laboral;

c) Cuestionarse el tipo de relación existente entre sí mismo/a y el entorno; es decir, tomar conciencia de los determinantes internos y externos que inciden sobre el propio desarrollo personal/profesional y el de la comunidad en la que se vive;

d) Tomar conciencia de los recursos, los medios, las oportunidades que existen para transformar la realidad.

El desarrollo de la conducta exploratoria se puede facilitar, según Blustein (1992), a través de las siguientes estrategias:

Aumentar el sentido del control de la persona que se orienta.

Incrementar sus competencias para su propia autoexploración.

Mejorar las competencias de exploración del entorno.

Ayudarles a experimentar un nivel adaptativo de 'inmanencia'.

Enseñarles a ser objetivos en el procesamiento de la información.

Modelar actitudes y comportamientos efectivos de exploración.

Ayudarles a evitar conclusiones prematuras. 
Como se puede observar, lo más importante del aprendizaje de la conducta exploratoria no es tanto el contenido, la información en torno a la cual se aplica la misma, como el propio desarrollo del proceso de indagación. Este proceso se llevará a cabo en torno a dos tipos de contenidos:

a) Exploración de sí mismo/a;

b) Exploración del entorno.

Se presentan a continuación los aspectos específicos a trabajar en cada uno de estos bloques de contenido.

\section{Exploración de sí mismo/a}

Los aspectos en torno a los cuales puede girar la exploración sobre sí mismo/a son los siguientes:

Exploración de Sí mismo/a $\left\{\begin{array}{l}\text { Valores profesionales } \\ \text { Historia de vida }\left\{\begin{array}{l}\text { Género } \\ \text { Clase social } \\ \text { Proc. Cultural }\end{array}\right. \\ \text { Intereses profesionales } \\ \text { Sueños/aspiraciones } \\ \text { Motivación } \\ \text { Competencias profesionales y formación } \\ \text { Estilos de aprendizaje }\end{array}\right.$

- La exploración de los valores profesionales tiene interés por cuanto son los que fundamentan las decisiones profesionales. Los valores son la consecuencia de la conjunción de variables personales (sexo, edad, procedencia social o cultural) y situacionales (situación sociolaboral general y personal, situación familiar, valores culturales...). En los valores profesionales se da un cotinuum que va desde la consideración del trabajo como fuente de realización personal (perspectiva expresiva), hasta la valoración del trabajo sólo como fuente de ingresos materiales (perspectiva instrumental). El posicionamiento de una persona ante dichos valores no es siempre el mismo: depende del momento, la situación personal e incluso del tipo de trabajo que se esté realizando.

- El cuestionamiento de la propia historia de vida conlleva un análisis crítico de la incidencia que han tenido, tienen y pueden tener en un futuro, determinadas variables sobre el propio desarrollo personal y profesional. Estas variables hacen referencia, entre otras, al género y la procedencia social y cultural.

- Los intereses profesionales, al igual que los valores, participan en el proceso de toma de decisiones profesionales. Super (1995) los define como las actividades a través de las cuales la persona espera llevar a la práctica sus valores para, así, satisfacer sus necesidades. 
- Los sueños y las aspiraciones, que actúan como generadores de representaciones de nuevas realidades y, por tanto, como elementos de superación de estereotipos y determinismos.

- La motivación, que actúa como motor para iniciar el proceso de exploración de sí mismo/a y del entorno, para poder determinar lo que se es y lo que se desea ser, elementos básicos para construir los proyectos profesionales y vitales.

- Las competencias profesionales y la formación. El balance de competencias profesionales permite a la persona tomar conciencia de sus posibilidades y de los aspectos que aún le quedan por desarrollar. Facilita tanto el propio "marketing" ante los/as posibles empleadores/as como la elaboración de itinerarios formativos.

- El interés de la exploración de los estilos de aprendizaje reside en su potencialidad para facilitar los procesos de formación (inicial, continua) de la persona que se orienta.

Exploración del entorno

En relación a este aspecto se deben realizar algunas apreciaciones:

a) Necesidad de aprender a buscar, valorar críticamente la información y traducirla a conocimiento útil;

b) La exploración debe estar abierta a formas no tradicionales o típicas de trabajo;

c) Las prácticas/practicum deben y pueden tener un valor no sólo formativo, sino también orientador;

d) Se debe facilitar el ejercicio de la imaginación y la toma de conciencia crítica de las paradojas sociolaborales.

Algunos de los aspectos a explorar son los siguientes:

\begin{tabular}{|c|c|}
\hline Inform. Académica & $\begin{array}{l}\text { Oportunidades educativas (ofertas curriculares y no-curriculares) } \\
\text { Exigencias académicas } \\
\text { Requisitos ingreso }\end{array}$ \\
\hline $\begin{array}{l}\text { Inform. Profes. y } \\
\text { ocupacional }\end{array}$ & $\begin{array}{l}\text { Características puestos de trabajo } \\
\text { Requisitos admisión } \\
\text { Condiciones de trabajo } \\
\text { Fuentes de información } \\
\text { Situación mercado laboral } \\
\text { Estructura profesiones } \\
\text { Forma de trabajo (remunerado y no remun.) }\end{array}$ \\
\hline Comunidad & $\begin{array}{l}\text { Situación y organización geográfica } \\
\text { Antecedentes históricos } \\
\text { Proceso de cambio cultural } \\
\text { Característica de la población } \\
\text { Organización social } \\
\text { Condiciones económicas } \\
\text { Características políticas }\end{array}$ \\
\hline
\end{tabular}


El desarrollo de la conducta exploratoria en relación al entorno implica, por tanto, la recogida, valoración, análisis crítico y utilización de información profesional-ocupacional (donde se incluyen experiencias de acercamiento al entorno de trabajo), académica y sobre la comunidad. En el proceso a desarrollar no es tan importante el producto de la información - aunque debe ser de calidad - como el aprendizaje del proceso, transferible a situaciones futuras.

\section{Construcción de la identidad personal y colectiva: cristalización}

En el aprendizaje de la tarea de cristalización está implicado el desarrollo de habilidades y actitudes como la reducción, clasificación, resumen, organización, coherencia... La puesta en práctica de estas habilidades y actitudes debe facilitar la construcción de la propia identidad personal-profesional y la determinación de preferencias en relación con determinados campos y estilos profesionales y de vida. Asimismo, debe favorecer el desarrollo de la identidad como grupo.

Los aspectos implicados en el desarrollo de la tarea de cristalización son los siguientes:

$$
\begin{aligned}
& \text { Identidad personal-profesional } \\
& \text { Identidad grupal } \\
& \text { Roles profesionales vs. roles vitales } \\
& \text { Significado del trabajo }
\end{aligned}
$$

- Identidad personal-profesional: se constituye como el hilo conductor de la trayectoria vital en una situación de caos, movilidad, flexibilidad, inestabilidad e incertidumbre. Facilita enfrentarse a la complejidad externa de forma menos traumática. Para construirla no es suficiente con recoger suficiente información sobre sí mismo/a y el entorno, sino aprender a dar un significado personal a dicha información. Esta construcción facilita, en relación al propio proceso de orientación:

a) Conocer lo que se puede llegar a ser: Facilita el posterior desarrollo de competencias relacionadas con el saber-ser y el saber-estar;

b) Mejorar la autoestima;

c) Potenciar habilidades y destrezas para comprender el propio desarrollo;

d) Fomentar el equilibrio personal en la conjugación de los diferentes roles vitales;

e) Actuar de forma coherente;

f) Tomar conciencia de sí mismo/a como principal agente de la propia vida, lo cual facilita:

- el acceso al mercado laboral a corto plazo;

- el desarrollo de competencias útiles para mantenerse en el puesto de trabajo a largo plazo;

- el desarrollo de una mayor satisfacción personal. 
- La identidad grupal o colectiva: significa, según De la Riva (1998) aprender a pensar, sentir, asociarse y actuar como grupo. Se desarrolla a partir del intercambio grupal de conocimientos y experiencias previas y es elemento básico para promover transformaciones sociales.

- Roles profesionales y su relación con los roles vitales: se plantea la necesidad de aprender a conceptuar los roles que se desempeñan o se desean desarrollar así como a resolver los conflictos que se producen entre distintos roles y a superar los sentimientos que se generan como consecuencia de dichas tensiones. Se debe considerar que los procesos de toma de decisiones se producen cuando una persona asume o anticipa que va a asumir un nuevo rol.

- Significado del trabajo: es la importancia que atribuye la persona al hecho de trabajar. Esta atribución va a depender tanto de las situaciones en las que se desarrollo el trabajo como de la forma personal que cada individuo tenga de vivir e interpretar dichas situaciones.

\section{Aprender a construir proyectos}

Los conocimientos, habilidades y actitudes vinculadas a este aprendizaje están relacionados con las tareas de especificación y de realización. Si bien la construcción de proyectos profesionales implica todo el proceso de exploración-cristalización-especificación-realización, su aprendizaje está especialmente vinculado a las dos últimas tareas, compartiendo la última (realización) con el "aprender a actuar". En el cuadro 2 se recogen algunas de las habilidades y actitudes que deben ser desarrolladas para dominar ambas tareas:

\section{CUADRO 2: Habilidades y actitudes implicadas en la construcción de proyectos profesionales y vitales (adaptado de Pelletier y Bujold, 1984).}

\begin{tabular}{|c|l|l|}
\hline $\begin{array}{c}\text { Tareas de desarrollo } \\
\text { vocacional }\end{array}$ & \multicolumn{1}{|c|}{ Habilidades } & \multicolumn{1}{c|}{ Actitudes } \\
\hline Especificación & $\begin{array}{l}\text { Comparar } \\
\text { Jerarquizar } \\
\text { Eliminar } \\
\text { Elegir } \\
\text { Examinar } \\
\text { Evaluar }\end{array}$ & $\begin{array}{l}\text { Apreciación } \\
\text { Confianza } \\
\text { Responsabilidad } \\
\text { Discriminación } \\
\text { Sentido crítico } \\
\text { Tendencia a la reflexión }\end{array}$ \\
\hline \multirow{2}{*}{ Realización } & Deducir & Certeza \\
& $\begin{array}{l}\text { Prever } \\
\text { Aplicar } \\
\text { Generalizar } \\
\text { Planificar } \\
\text { Elaborar }\end{array}$ & $\begin{array}{l}\text { Determinación } \\
\text { Perspicacia } \\
\text { Sentido práctico }\end{array}$ \\
\hline
\end{tabular}

La elaboración de proyectos profesionales en sí misma, implica una serie de aprendizajes que se desarrollan a continuación: 
Aprender a tomar decisiones

Contrastar lo deseable con lo probable

Autenticidad

Sentido crítico

Realismo

Autocrítica

Descubrir nuevas formas de acción

Aprender a elaborar proyectos $\begin{cases}\text { Elaboración } & \begin{array}{l}\text { Proyección } \\ \text { Planificación }\end{array} \\ \text { Extrapolación } & \left\{\begin{array}{l}\text { Previsión } \\ \text { Extrategia }\end{array}\right.\end{cases}$

Aprender a organizarse

Aprender a tomar decisiones (especificación)

Aprender a tomar decisiones implica:

a) Contrastar lo deseable con lo probable;

b) Identificar las motivaciones que subyacen a los propios comportamientos (autenticidad);

c) Valorar críticamente las informaciones que se poseen (sentido crítico);

d) Tomar conciencia de la necesidad de buscar el equilibrio entre deseos y posibilidades (individuales y/o colectivas) (realismo);

e) Tomar conciencia de sí mismo/a y/o el grupo, con las contradicciones y conflictos que todas las personas llevan consigo mismas (autocrítica);

f) Afrontar los sentimientos (incertidumbre, ansiedad) que van emparejados a cualquier situación de toma de decisiones;

g) Aprender a construir nuevas formas e ideas de acción colectiva.

En el desarrollo de este aprendizaje se debe considerar:

- Que existen diferentes estilos de toma de decisiones (activo/pasivo; implicado/dependiente; emocional/racional).

- Que existen situaciones de indecisión profesional propias del mismo proceso de toma de decisiones, aunque se debe evitar que se conviertan en permanentes.

- Que las decisiones están sujetas a evolución y, por tanto, son provisionales.

- Que lo más importante es el aprendizaje del proceso de toma de decisiones, por encima del producto del mismo.

\section{Elaboración del proyecto en sí mismo (realización)}

Las actividades que están implicadas en el proceso de planificación de la acción a emprender son las siguientes (aplicables al individuo o al grupo): 
- Descripción detallada del plan de acción a llevar a cabo (elaboración). A su vez, implica:

a) Análisis del sentido que puede tener la decisión adoptada — provisionalmenteen un futuro inmediato, de las consecuencias que puede tener en la vida cotidiana (proyección);

b) Búsqueda de fuentes de información que ayuden a tomar conciencia de las consecuencias que pueden tener las elecciones y de los pasos a dar para llevarlas a cabo (planificación).

- Previsión de las consecuencias que pueden tener en un futuro las acciones y situaciones presentes (extrapolación):

a) Análisis de los riesgos y posibilidades de éxito del proyecto (previsión);

b) Prepararse para afrontar dificultades y superar obstáculos (a nivel práctico y afectivo-emocional) (estrategia).

- Aprender a organizarse como grupo.

\section{Aprender a actuar}

Este aprendizaje está directamente ligado al desarrollo de la tarea de realización e implica el desarrollo de los conocimientos, habilidades y actitudes que facilitan la estructuración y organización de un proyecto colectivo de transformación de la realidad. Asimismo, implica el aprendizaje de los procesos y estrategias relacionadas con la empleabilidad y la ocupabilidad.

$$
\begin{aligned}
& \text { Empleabilidad } \\
& \text { Ocupabilidad } \\
& \text { Competencias participativas } \\
& \text { Planificación del ocio y el tiempo libre }
\end{aligned}
$$

\section{Empleabilidad}

Se entiende como la capacidad para insertarse en el empleo. Los contenidos que están implicados en este aprendizaje son los siguientes:

a) Variables personales: Currículo; capacidad de aprendizaje; actitudes de inserción; autoestima profesional; disponibilidad laboral

b) Técnicas y estrategias de inserción: planificación de la búsqueda de empleo; pasos para determinar las ocupaciones adecuadas para cada persona; canales de información para hacer la oferta; técnicas de búsqueda de empleo; espíritu emprendedor; estrategias de autoempleo; creación de cooperativas, sociedades empresariales; creación de asociaciones profesionales. 


\section{Ocupabilidad}

Es la capacidad para adaptarse a las nuevas exigencias requeridas en el mercado de trabajo e implica los siguientes aprendizajes:

- Preparación para la movilidad geográfica y cultural. La movilidad facilita: inserción laboral; desarrollo de la carrera (promoción) en el interior de las organizaciones; incremento de opciones formativas.

- Desarrollo continuo de competencias profesionales, que se facilitará a través del desarrollo, desde los primeros años escolares del aprender a aprender.

\section{Desarrollo de competencias participativas}

Implica el desarrollo de proyectos colectivos de diversa índole: Colegios, asociaciones profesionales que permitan la generación, reivindicación, delimitación de campos y formas profesionales de hacer; proyectos de corte empresarial; organizaciones sin ánimo de lucro; colectivos de voluntariado, con valor en sí mismas

Desde una perspectiva crítica de la orientación se destaca la importancia del desarrollo del tercer sistema tal como lo describe Luque Domínguez (1995): proyectos comunitarios de interés social que están por encima de los intereses políticos o individuales. Se trata de buscar soluciones a los problemas de la sociedad civil desde entro, a través de la democracia participativa y crítica. Se trata, como indica Pérez Serrano (1997) de dotar de valores de libertad, autonomía, emancipación y solidaridad al proceso de toma de decisiones.

\section{Planificación del ocio y el tiempo libre}

Consiste en aprender a buscar el equilibrio (de calidad más que de cantidad) entre tiempo de trabajo y tiempo libre. Se entiende que trabajo, ocio y proyecto de vida están estrechamente interrelacionados: se trata de aprender a utilizar el tiempo libre de acuerdo con las potencialidades personales, con un estilo y proyecto de vida determinados.

\section{Para finalizar...}

Orientar a las personas para que aprendan a construir proyectos profesionales y vitales significa, como se ha podido observar, considerarlas actrices principales de sus correspondientes trayectorias vitales (personales y profesionales) y, por tanto, a ser más libres y autoras de sus propias vidas. Un reto difícil, pero apasionante para el/la profesional de la orientación.

\section{Referencias bibliográficas}

ADAPT. (1995). Projets des jeunes. Une question d'identité. Paris: ADAPT.

Alex, L. (1991): "Descripción y registro de las cualificaciones. El concepto de cualificación". Formación Profesional, 1, pp. 23-27. 
Arnold, J. y Jackson, J. C. (1997). "The new career: issues and challenges". British Journal of Guidance and Counselling, 25, pp. 427-433.

Ballion, R. (1986). Le transfert des acquis. Recherche-action des services d'Orientation engagés dans le dispositif d'insertion des jeunes. Paris: Ministère de l'Education Nationale.

Baptiste, A., \& Belisle, C. (1991). Dossier Photolangage. Paris: Les Éditions d'Organisation.

Baptiste, A., Belisle, C., Pechenart, J.-M., \& Vacheret, C. (1991). Photolangage.Un méthode pour communiquer en groupe par la photo. Paris: Les Ëditions d'Organisation.

Bernard, F. (1995). "Comment sortir du labyrinthe". Cahiers Pédagogiques, pp. 36-37.

Blustein, D. L. (1992). "Applying current theory and research in career exploration to practice". The Career Development Quarterly, 41, pp. 174-184.

Bonvalot, G. E. C. B. (1986). "Projet et autoformation professionnelle". Education Permanente, pp. 53-65.

Boursier, S. y Langlois, J.-M. (1993). L'orientation a-t-elle un sens? Paris: Entente.

Boutinet, J. P. (1990). Antropologie du projet. Paris: PUF.

Bunk, G. P. (1994). "Las transmisión de las competencias en la formación y el perfeccionamiento profesionales en la RFA". Revista Europea de Formación Profesional, 1, pp. 8-14.

Castells, M. (1994): Nuevas perspectives críticas en educación. Barcelona: Paidós.

Castells, M. (1996). La era de la información. Economía, sociedad y cultura. Vol. I: La sociedad red. Madrid: Alianza.

Caspar, P. (1994): "La inversió inmaterial”. Taleia, pp. 34-39.

Casullo, M. M., \& Cayssials, A. N. (1994). Proyecto de vida y decisión vocacional. Barcelona: Paidós.

CEE (1994): Crecimiento, competitividad y empleo. Luxemburgo: Oficina de Publicaciones Oficiales de las Comunidades Europeas.

CEE (1996): Libro Blanco Enseñar y Aprender en la Sociedad Cognitiva. Luxemburgo: Oficina de Publicaciones Oficiales de las Comunidades Europeas.

Centre for Guidance Studies. University of Derby. Reshaping career development for the 21 st century. ed. Derby.

Collin, A. (1997). "Career in context". British Journal of Guidance and Counselling, 25, pp. 435-446.

Collin, A. y Watts, A. G. (1996). "The death and transfiguration of career and of career guidance?". British Journal of Guidance and Counselling, 24, pp. 385-398.

Corominas, E., \& Isús, S. (1998). “Transiciones y orientación”. Revista de Investigación Educativa, 16, pp. $155-184$.

De la Riva Rodríguez, F. (1998). "Principios metodológicos para la formación en Educación Social". In G. Pérez Serrano (Ed.), Contexto cultural y socioeducativo de la educación social. (pp. 167204). Sevilla: Universidad de Sevilla.

Defrenne, R. (1995). "La place des valeurs dans la construction des projets". L'Indecis, pp. 74-77.

Defrenne, R. (1998). "Pour l'orientation et l'insertion, un nouvel horizon: l'approche expérientielle éducative et sociale". L'Indecis, pp. 113-128.

Delors, J. (1996). La educación encierra un tesoro. Madrid: Santillana-UNESCO.

Echeverría, B. (1993): Formación Profesional. Guía para el seguimiento de su evolución. Barcelona: PPU.

Echeverría, B. (1999). "Profesión, formación y orientación". En L. Sobrado (Ed.). Orientación e información sociolaboral. Barcelona: Estel (9-40). 
Fernández Sierra, J. (1998). “Orientación profesional: ¿hacia qué horizonte?”. In X. De Salvador \& M. L. Rodicio (Eds.), ¿Para onde camiña a orientación? (pp. 39-62). A Coruña: Universidade da Coruña.

Gallego Matas, S. (1999). Cómo planificar el desarrollo profesional. Actividades y estrategias de autoorientación. Barcelona: Laertes.

Giarini y Liedtke, P. M. (1998). El dilema del empleo. El futuro del trabajo. Barcelona: Galaxia-Gutemberg.

Guichard, J. (1995). La escuela y las representaciones de futuro de los adolescentes. Barcelona: Laertes.

Guichard, J. (1995). "Psychopedagogie du projet d'avenir et normativité". In ADAPT (Ed.), Projets des jeunes. Une question d'identité. (pp. 9-40). Paris: ADAPT.

Herr, E. L. (1996). "Perspectivas on ecological context, social policy, and career guidance". The Career Development Quarterly, pp. 5-19.

Herrera Menchén, M. M. (1998). El desarrollo de procesos de acción socioeducativa desde la perspectiva de la animación sociocultural. Sevilla: Universidad de Sevilla.

Lafont, M. C. (1995). "Monográfico sobre Le projet personnel de l'élève”. Cahiers Pedagogiques.

Latreille, G. (1984). Les chemins de l'orientation professionnelle. Lyon: Presses Universitaires de Lyon.

Latreille, G. (1995). "Les paradoxes du métier collectivement Trouver/Créer". L'Indecis, pp. 49-54.

Law, B. (1999). "Career learning space: new DOTS thinking for careers education”. British Journal of Guidance and Counselling, 27, pp. 35-54.

Legrés, J., \& Pémartin, D. (1988). Les projets chez les jeunes. La psychopédagogie des projets personnels. Paris: EAP.

Luque Domínguez, P. A. (1995). Espacios educativos. Sobre la transformación y la participación social. Barcelona: EUB.

Mitchel, K. E., Levin, A. S., \& Krumboltz, J. D. (1999). "Planned happenstance: constructing unexpected career opportunities". Journal of Counseling and Development, 77, pp. 115-124.

Mure, J. L. (1997). "La orientación: un proceso de desarrollo complejo y educativo en un mundo incierto". In Instituto Andaluz de la Mujer (Ed.), Programa Elige. Material de apoyo. (pp. 55-66). Sevilla: Autor.

Mure, J. L. (1997). "Le projet: une aspiration a être dans une monde complexe et uncertair". L'Indecis, pp. 29-35.

Pelletier, D. (1994). “Apprendre a choisir à l'Université”. L'Indecis, 7-11.

Pelletier, D. (1981). "L'approche opératoire du dévelopement personnel et voctionnel: ses fondements et ses valeurs". Revue PMS, pp. 3-22.

Pelletier, D. (1995). "Aproximación operativa del desarrollo personal y voacional: sus fundamentos y sus valores". Revista d'Orientació, VII, pp. 3-22.

Pelletier, D. (1986). "Le projet ou l'elaboration cognitive du besoin". Education Permanente, pp. 52-62.

Pelletier, D. (1984). Pour une approche éducative en orientation. Québec: Gäetan Morin.

Pérez Serrano, G. (1997). Cómo educar para la democracia. Estrategias educativas. Madrid: Popular.

Riverin-Simard, D. (1996). Transitions professionnelles: choix et strategies. Québec: Presses de l'Univesté de Laval. 
Rodríguez Moreno, M. L. (1999). Enseñar a explorar el mundo del trabajo. Diagnóstico de las destrezas exploratorias y propuestas de intervención. Archidona (Málaga): Aljibe.

Rodríguez Moreno, M. L. (1999). “La preparación para el trabajo: el proyecto profesional”. Revista d'Orientació, XII, pp. 5-22.

Rodríguez Moreno, M. L., \& Gallego Matas, S. (1999). "El proyecto profesional, herramienta de intervención en la función tutorial universitaria". Revista Española de Orientación y Psicopedagogía, 10, pp. 179-192.

Romero Rodríguez, S. (1999). Orientación para la transición de la escuela a la vida activa. Barcelona: Laertes.

Romero Rodríguez, S. (1998). "Una metodología de orientación como proceso de desarrollo personal y social: hacia una orientación desde la diferencia sexual". In Instituto Navarro de la Mujer (Ed.), Hacia una orientación profesional no secista. (pp. 89-113). Pamplona: Publicaciones del Gobierno de Navarra.

Savickas, M. (1993). "Career counselling in the postmodern era". Journal of Cognitive Psychotherapy, pp. 205-215.

Solazzi, R. (1993). "De l'ADVP á un nouvelle approche en orientation”. L'Indecis, pp. 20-30.

Solazzi, R. (1997). "Paradoxes en orientation”. L'Indecis, pp. 37-70.

Super, D. E. (1990). "A life-span, life-space approach to career development”. En D. Brown y L. Brooks (Eds.). Career choice and development. San Francisco: Jossey-Bass. (197-261).

Super, D. E. (1995). "Models of career development". In AIOSP (Ed.), Actas de la conferencia Internacional de IAEVG: Career Guidance Services for the 90's. (pp. 47-66). Lisboa: Autor.

Vondraceck, F. W. y col. (1986). Career development: a life-span developmental approach. Hillsdale, N. J.: Lawrence Erlbaun Ass. Pub.

Young, R. A. (1984). "Toward an ecology of career development". Canadian Counselor, pp. 152-159.

Young, R. A.; Valach, L. y Collin, A. (1996). "A contextual explanation of career”. En D. Brown y L. Brooks (Eds.). Career choice and development. San Francisco: Jossey Bass.

Zuazua, A. (1997). "Valores de la psicología humanista y de la teoría crítica en la reforma educativa". Revista de Orientación y Psicopedagogía, 8, pp. 77-92.

Fecha de recepción: 19-04-2003

Fecha de revisión: 29-09-2003

Fecha de aceptación: 05-11-2004 